Kragujevac Journal of Mathematics

Volume 45(4) (2021), Pages 607-613.

\title{
ON CO-FILTERS IN SEMIGROUPS WITH APARTNESS
}

\author{
DANIEL A. ROMANO ${ }^{1}$
}

\begin{abstract}
The logical environment of this research is the Intuitionistic Logic and principled-philosophical orientation of the Bishop's Constructive Mathematics. In this paper, basing our consideration on the sets with the apartness relation, we analyze the lattices of all co-filters of an ordered semigroup under a co-quasiorder as a continuation of our article [19]. We prove a number of results related to co-filters in a semigroup with apartness and the lattice of all co-filters of such semigroups.
\end{abstract}

\section{INTRODUCTION}

The setting of this research is Bishop's constructive mathematics [2-5,11,20], mathematics developed on the Intuitionistic logic [20]. In our text [19] we talked about co-ideals and co-filters in sets with apartness ordered under a co-quasiorder relation (co-order relation). In this text, the word will be about the co-filters of the semigroups with apartness ordered under a co-quasiorder relation (co-order relation).

We refer the reader to look at our previously published texts $[6,7,12,16,18]$ for more details on semigroups with apartness. In these articles, the concept of co-order relations and the concept of co-quasiorder relations in such semigroups have been introduced and analyzed. Additionally, these relations are left and right cancellative with respect to apartness.

In this text, we are interested in the left and right classes of co-quasiorder (co-order) relation generated by a subset of a semigroup with apartness ordered under the coquasiorder (co-order) relation. Concepts of co-quasiorder and co-order on sets with apartness are investigated by this author in many of his articles. (See for example $[14-18])$.

Key words and phrases. Bishop's constructive mathematics, semigroup with apartness, co-order and co-quasiorder relations, co-filters.

2010 Mathematics Subject Classification. Primary: 03F65. Secondary: 20M99, 06F05, 06 D99.

DOI 10.46793/KgJMat2104.607R

Received: February 12, 2018.

Accepted: March 29, 2019. 
Let $(S,=, \neq)$ be a set with apartness. Any strongly extensional total function

$$
w: S \times S \ni(x, y) \mapsto x y \in S
$$

is an internal binary operation in $S$. If we are speaking in the language of classical algebra, it can be said that the function $w$ is left and right cancellative with respect to apartness relation

$$
(\forall x, y, z \in S)((x z \neq y z \vee z x \neq z y) \Rightarrow x \neq y) .
$$

System $S=((S,=, \neq), w)$ is called a grupoid with apartness. Additionally, if the operation $w$ is associative, then the system $S$ is a semigrop with apartness. A relation $\not$ in $S$ is a co-quasiorder relation (co-order relation) in $S$ if it is consistent $\Varangle \subseteq \neq$,

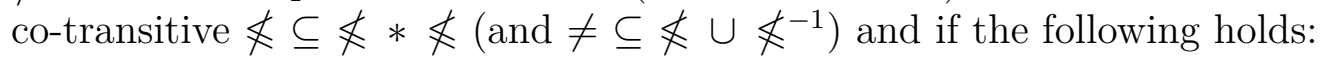

$$
(\forall x, y, z \in S)((x z \nless y z \vee z x \nless z y) \Rightarrow x \nless y) \text {. }
$$

In this text we will accept the following assumption

$$
(\forall x, y \in S)(\neg(x \nless x y) \wedge \neg(y \nless x y)) .
$$

The usual term used to indicate this kind of relations is that the relation ' $\nless$ ' is a negatively defined ordered relation [10].

\section{Co-Filters of Semigroups with Apartness}

Juhasz and Vernitski [10] expressed a statement: "There was no systematic study of filters in semigroups". By reviewing the available literature on the internet, we found a very small number of texts in which the filters were researched in ordered semigroups: for example $[1,9,10]$. In older semigroup theory literature, filters (also known as faces and under several other names) were introduced as subsemigroups whose complement is an ideal. Some results concerning such filters were obtained in the 1960s and 1970s, see, for instance, $[1,8]$. Our intention is to introduce and analyze the concept of co-filters in semigroup with apartness ordered under a co-quasiorder (co-order). The concept of co-filters as a substructure in such semigroups is a dual of the concept of filters in the classical theory of ordered semigroups.

We will start this section with the following statement.

Proposition 2.1. Let $\$$ be a co-quasiorder on a semigroup $S$. Then the left class $L(a)$ and the right class $R(b)$ are strongly extensional subsets of $S$ such that $a \triangleright L(a)$ and $b \triangleright R(b)$ for any $a, b \in S$. Moreover, the following implications hold:

(0) classes $L(a)$ and $R(b)$ are co-subsemigroups of semigroup $S$;

(1) $y \in L(a) \wedge x \in S \Rightarrow x \in L(a) \vee x \nless y$;

(2) $y \in R(b) \wedge x \in S \Rightarrow x \in R(b) \vee y \nless x$;

(3) $a \nless b \Rightarrow L(a) \cup R(b)=S$;

(4) $a \neq b \Rightarrow b \in L(a) \vee a \in R(b)$ if $\$$ is an co-order relation. 
Proof. For illustration we will show how some of these claims are proven. Other assertions are proved by analogy.

Let $x \in S$ and $y \in L(a)$. Then, by co-transitivity of $\nless$, we have $a \nless x$ or $x \nless y$. So, $x \in L(a)$ or, by consistency of $\nless, x \neq y$. Thus, $L(a)$ is a strongly extensional subset of $S$ and $a \triangleright L(a)$ holds.

(0) The subset $L(a)$ is a co-subsemigroup of semigroup $S$. Indeed, suppose $x, y \in$ $L(a)$. Then from $a \nless x y$ follows $a \nless x$ or $x \nless x y$. Thus $x \in L(a)$ by hypothesis (1.1). Also, from $a \nless x y$ we have $a \nless y$ or $y \nless x y$. Thus $y \in L(a)$ by hypothesis (1.1) too.

(1) From $y \in L(a) \wedge x \in S$, i.e., from $a \nless y \wedge x \in S$ we have $a \nless x \vee x \nless y$. Then $x \in L(a) \vee x \notin y$.

In a similar manner we can prove that $R(b)$ is a strongly extensional subsemigroup of $S$ with $b \triangleright R(b)$ and that the implication $y \in R(b) \wedge x \in S \Rightarrow x \in R(b) \vee y \nless x$ holds.

The concept of co-filters in an ordered semigroup $S$ is introduced by the following definition.

Definition 2.1. For subset $G$ of $S$ we say that it is a co-filter in $S$ if

and

$$
(\forall x, y \in S)(x y \in G \Rightarrow(x \in G \vee y \in G))
$$

$$
(\forall x, y \in S)(y \in G \wedge x \in S \Rightarrow(x \in G \vee x \nless y)) .
$$

According to the first property, the co-filter is a co-subsemigroup in a semigroup $S$. From another property, immediately follows that a co-filter in semigroup $\mathrm{S}$ is a strongly extensional subset in S. So, the subset $L(a)$ is a principal co-filter of $S$ generated by the element $a$. In addition, the sets $\emptyset$ and $S$ are trivial co-filters of $S$.

Remark 2.1. Since $\$$ is a negatively defined co-quasiorder in $S$, for any co-filter $G$ in $S$ we have $x y \in G \Rightarrow(x \in G \wedge y \in G)$. Indeed. Let for elements $x$ and $y$ holds $x y \in G$. Thus $x y \in G \Rightarrow(x \in G \vee x \nless x y)$ and $x y \in G \Rightarrow(y \in G \vee y \nless x y)$. According the hypothesis (1.1), we finally have $x \in G$ and $y \in G$.

In the following statement we show that a strongly complement of a co-filter is a filter.

Theorem 2.1. If $G$ is a co-filter of ordered semigroup $S$, then $G^{\triangleright}$ is a filter in ordered semigroup $S$ under quasi-order $\mathbb{Z}^{\triangleright}$.

Proof. Let $x \in G^{\triangleright}$ and $y \in G^{\triangleright}$ and let $u$ be an arbitrary element in $G$. By strongly extensionality of $G$ follows $u \neq x y$ or $x y \in G$. Since the second option leads to contradiction, we conclude $x y \neq u \in G$. So, the subset $G^{\triangleright}$ is a subsemigroup in $S$.

Let $G$ be a co-filter of $S$. Then ${ }^{\triangleright}$ is a quasi-order on semigroup $S$. Suppose that $x \in G^{\triangleleft}$ and $x \Varangle^{\triangleleft} y$. Let $u$ be an arbitrary element of $G$. Thus, from the implication $u \in G \Rightarrow x \in G \vee x \nless u$ follows $x \nless u$ because $x \triangleright G$. Further on, by co-transitivity 
of $\Varangle$, we have $x \nless y \vee y \nless u$. Hence, we conclude $y \neq u \in G$ because $x \Varangle^{\triangleright} y$. Finally, $y \in G^{\triangleright}$. So, the subset $G^{\triangleright}$ is a filter in $S$.

Theorem 2.2. Let $f:\left(S, \Varangle_{S}\right) \Rightarrow\left(T, \$_{T}\right)$ be a reverse isotone homomorphism between two ordered semigroups with apartness under co-quasiordereds. If $G$ is a co-filter in $T$, then the set $f^{-1}(G)=\{a \in S: f(a) \in G\}$ is a co-filter in $S$.

Proof. Let $x, y \in S$ be arbitrary elements such that $x y \in f^{-1}(G)$. Then $f(x y) \in G$ and $f(x) f(y) \in G$. Thus $f(x) \in G$ or $f(y) \in G$. Therefore, $x \in f^{-1}(G)$ or $y \in f^{-1}(G)$ and the subset $f^{-1}(G)$ is a cosubsemigroup of semigroup $S$.

Let $y \in f^{-1}(G)$ and $x \in S$ be arbitrary elements. Thus, $f(y) \in G$ and $f(x) \in T$. Hence $f(x) \in G$ or $f(x) \Varangle_{T} f(y)$. Therefore, we have $x \in f^{-1}(G)$ or $x \Varangle_{S} y$ because $f$ is a reverse isotone homomorphism.

Finally, the subset $f^{-1}(G)$ is a co-filter in $S$.

In following text, we represent some properties of the union of co-filters. In the following theorem, we prove that the union of any family of co-filters is a co-filter again.

Theorem 2.3. If $\left\{G_{j}\right\}_{j \in J}$ be a family of co-filters in $S$, then $\bigcup_{j \in J} G_{j}$ is a co-filter too.

Proof. If $x y \in \cup_{j \in J} G_{j}$, then there exists an index $j \in J$ such that $x y \in G_{j}$. Thus $x \in G_{j}$ or $y \in G_{j}$. So, $x \in \cup_{j \in J} G_{j}$ or $y \in \cup_{j \in J} G_{j}$. Therefore, $\cup_{j \in J} G_{j}$ is a cosubsemigroup in $S$. Let $y \in \bigcup_{j \in J} G_{j}$ and $x \in S$. Thus, there exists an index $j \in J$ such that $y \in G_{j}$. Hence, by definition of co-filter, we have $x \in G_{j}$ or $x \nless y$. Finally, we conclude $x \in \bigcup_{j \in J} G_{j}$ or $x \nless y$. Therefore, the union $\bigcup_{j \in J} G_{j}$ is a co-filter in $S$ too.

Corollary 2.1. Let $S$ is an ordered semigroup with apartness under co-quasiorder $\Varangle$. Then the family $\mathfrak{G}_{S}$ of all co-filters in $S$ forms a join semi-lattice. The greatest element in this semi-lattice is $S$.

Let $T$ be a subset of a semigroup $S$. Then, by previous theorem, $T^{R}=\bigcup_{t \in T} L(t)$ is a co-filter in $S$.

Definition 2.2. For a subset $T$ of a semigroup $S$ the co-filter $T^{R}$ is called ordered co-filter generated by subset $T$.

Particularly, for each element $a \in S$ the set $\{a\}^{R}$ is the principal ordered co-filter generated by element $a$ and, in addition, $\{a\}^{R}=L(a)$ holds.

Theorem 2.4. If $\left\{G_{j}\right\}_{j \in J}$ be a family of ordered co-filters in semigroup $S$, then $\cup_{j \in J} G_{j}$ is an ordered co-filter too.

Proof. Let $\left\{G_{j}\right\}_{j \in J}$ be a family of ordered co-filters in semigroup $S$. Then for any $j \in J$ there exists a subset $T_{j}$ of $S$ such that $G_{j}=T_{j}^{R}$. Since $\left(\bigcup_{j \in J} T_{j}\right)^{R}=\bigcup_{j \in J} T_{j}^{R}$ holds, it is directly verified that $\bigcup_{j \in J} G_{j}$ is an ordered co-filter in $S$ generated by subset $\bigcup_{j \in J} T_{j}$. 
Corollary 2.2. The family $\mathfrak{O}_{S}$ of all ordered co-filters form join semi-lattice.

In what follows we will represent our findings concerning the intersection of co-filters in semigroup with apartness.

Theorem 2.5. If $G_{1}$ and $G_{2}$ are co-filters in a semigroup $S$, then the intersection $G_{1} \cap G_{2}$ is also co-filter in $S$.

Proof. Let $x$ and $y$ be arbitrary element of $S$ such that $x y \in G_{1} \cap G_{2}$. It means $x y \in G_{1}$ and $x y \in G_{2}$. Thus $x \in G_{1} \wedge y \in G_{1}$ and $x \in G_{2} \wedge y \in G_{2}$ by Remark 2.1. So, we have $x \in G_{1} \cap G_{2} \wedge y \in G_{1} \cap G_{2}$. Therefore, the intersection $G_{1} \cap G_{2}$ is a co-subsemigroup in $S$.

From $y \in G_{1} \cap G_{2}$ and $x \in S$, i.e., from $y \in G_{1} \wedge y \in G_{2} \wedge x \in S$ follows $x \in G_{1} \vee x \nless y$ and $x \in G_{2} \vee x \nless y$. Thus, $x \in G_{1} \cap G_{2} \vee x \nless y$.

So, the intersection $G_{1} \cap G_{2}$ is a co-filter in $S$.

Corollary 2.3. The family $\mathfrak{G}_{S}$ of all co-filters in $S$ forms a lattice. The smallest and the greatest elements in this lattice are the empty set $\emptyset$ and $S$.

Remark 2.2. Let us note that if $G_{1}$ and $G_{2}$ be two order co-filters, than the intersection $G_{1} \cap G_{2}$ is not an ordered co-filter in general case. For example, the intersection of two ordered co-filters $G_{1}=A^{R}$ and $G_{2}=B^{R}$ is an ordered co-filter if the following holds

$$
(\forall a \in A)(\forall b \in B)(\exists c \in A \cap B)\left(c \mathbb{Z}^{\triangleright} a \wedge c \mathbb{Z}^{\triangleright} b\right) .
$$

Indeed, for arbitrary elements $y \in A^{R} \cap B^{R}$ and $x \in S$ there exist elements $a \in A$ and $b \in B$ such that $y \nless a$ and $y \nless b$. There exists an element $c \in A \cap B$ such that $c \Varangle^{\triangleright} a$ and $c \Varangle^{\triangleright} b$ by hypothesis. Thus, we have $y \nless c$. Further, from this follows $y \nless x$ or $x \nless c$ and finally we have $y \nless x$ or $x \in(A \cap B)^{R}$.

The previous analysis is the motivation for the introduction of the following definition.

Definition 2.3. An ordered semigroup $S$ is called directed if the following holds

$$
(\forall a, b \in S)(\exists c \in S)\left(c \mathbb{Z}^{\triangleright} a \wedge c \mathbb{Z}^{\triangleright} b\right) .
$$

Corollary 2.4. The family $\mathfrak{O}_{S}$ of all ordered co-filters in directed semigroup $S$ forms lattice.

Proof. Let $a$ and $b$ be arbitrary elements of semigroup $S$. Then there exists an element $c \in S$ such that $c \mathbb{Z}^{\triangleright} a$ and $c \mathbb{Z}^{\triangleright} b$. Thus, $L(c) \subseteq L(a) \cap L(b)$. Indeed. Suppose $c \notin s$. Thus $c \notin a \vee a \nless s$ and $c \notin b \vee b \nless s$. Then $a \nless s$ and $b \nless s$. Therefore, $L(c) \subseteq L(a) \cap L(b)$.

Corollary 2.5. The family of all principal co-filters in directed band $S$ forms lattice. Every finitely generated ordered co-filter is a principal co-filter. 
Proof. In a directed semigroup $S$ for any elements $a$ and $b$ there exists an element $c \in S$ such that $L(c) \subseteq L(a) \cap L(b)$, by previous corollary.

Since ' $\$$ is a negatively defined relation in semigroup $S$ with apartness, we have $L(a) \cup L(b) \subseteq L(a b)$ for any elements $a, b \in S$. Let $s$ be element in $S$ such

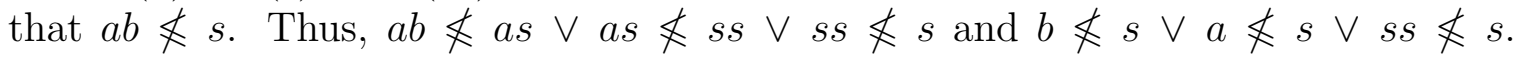
Therefore, $b \nless s \vee a \nless s$ because $s=s s$. Finally, we have $L(a b) \subseteq L(a) \cup L(b)$ and $L(a b)=L(a) \cup L(b)$.

As it has already been said, for each element $a \in S$ the set $\{a\}_{R}=L(a)$ is the principal co-filter generated by $a$. If $T$ is a finite set then, by Theorem 2.5, $T_{R}=\bigcap_{a \in A} L(a)$ is a co-filter in $S$ also. This is the motive to introduce the following concept.

Definition 2.4. Let $T \subseteq S$ be a finite subset of semigroup with apartness. Subsets of the form $T_{R}=\{z \in S:(\forall t \in T)(t \nless z)\}=\bigcap_{a \in A} L(a)$ are a normal co-filter in $S$.

Remark 2.3. Let $G$ be a normal co-filter of semigroup $S$ ordered under co-quasiorder $\Varangle$. Then there exists a finitely subset $T$ of $S$ such that $G=T_{R}$. If $z$ is an arbitrary element of $G$, we have $(\forall t \in T)(z \nless t)$ and $z \triangleright T$ because $\nless$ is a consistent relation. So, we have $(\forall z \in G)(z \triangleright T)$.

Theorem 2.6. If $\left\{G_{j}\right\}_{j \in J}$ is a finitely family of normal co-filters in semigroup $S$, then $\bigcap_{j \in J} G_{j}$ is a normal co-filter too.

Proof. Let $\left\{G_{j}\right\}_{j \in J}$ be a finitely family of normal co-filters in semigroup $S$. Then for each $j \in J$ there exists a subset $T_{j}$ of $S$ such that $G_{j}=\left(T_{j}\right)_{R}$. Since $\bigcap_{j \in J}\left(T_{j}\right)_{R}=$ $\left(\cup_{j \in J} R_{j}\right)_{R}$ holds, we conclude that the intersection $\bigcap_{j \in J} G_{j}$ is a normal co-filter of $S$.

Corollary 2.6. The family $\mathfrak{N}_{\mathfrak{S}}$ of all normal co-filters forms meet semi-lattice.

Final observation. As one of the answers to the question: "Why should the content of this text be mathematically acceptable?" we can offer the next reflection. Why is a text that contains thoughts about some algebraic concepts acceptable by the mathematical community? According to the usual standards, the text is mathematically acceptable because the author(s) expound and prove in an acceptable way some new logical possibility of the mentioned algebraic objects. To the first question raised above, we need to offer a completely identical answer according to the usual standards, by our opinion.

Why should this be interesting for a significant number of mathematicians? This is another question that naturally appears. Well, it does not need to be. The questions and answers to the observation and expression of logical possibilities in the constructive algebra are interesting only to interested logicians and mathematicians.

Many aspects of constructive mathematics are not just logical hygiene: avoid indirect proofs in favor of explicit constructions, detect and eliminate needless uses of the axiom of choice and so on. Of course, constructivism goes deeper than that. 
By accepting the non-existence of the TND principle, it is possible to have the multilayered properties of algebraic objects and processes with them. In this article, this two-stratification is shown on the example of filters and co-filters in semi-groups with apartness.

\section{REFERENCES}

[1] C. E. Aull, Ideals and filters, Compos. Math. 18(1-2) (1967), 79-86.

[2] E. Bishop, Foundations of Constructive Analysis, McGraw-Hill, New York, 1967.

[3] E. Bishop and D. Bridges, Constructive Analysis, Grundlehren der Mathematischen Wissenschaften 279, Springer, Berlin, 1985.

[4] D. Bridges and F. Richman, Varieties of Constructive Mathematics, London Mathematical Society Lecture Notes 97, Cambridge University Press, Cambridge, 1987.

[5] D. S. Bridges and L. S. Vita, Apartness and Uniformity: A Constructive Development, CiE series - Theory and Applications of Computability, Springer Verlag, Berlin, Heidelberg, 2011.

[6] S. Crvenković, M. Mitrović and D. A. Romano, Semigroups with Apartness, Mathematical Logic $\mathbf{5 9}(6)$ (2013), 407-414.

[7] S. Crvenković, M. Mitrović and D. A. Romano, Basic notions of (constructive) semigroups with apartness, Semigroup Forum 92(3) (2016), 659-674.

[8] O. Frink and R. S. Smith, On the distributivity of the lattice of filters of a groupoid, Pac. J. Math. Ind. 42 (1972), 313-322.

[9] J. Jakubak, On filters of ordered semigroups, Czechoslovak Math. J. 43(3) (1993), 519-522.

[10] Z. Juhasz and A. Vernitski, Filters in (quasiordered) semigroups and lattices of filters, Communication in Algebra 39(11) (2011), 4319-4335.

[11] R. Mines, F. Richman and W. Ruitenburg, A Course of Constructive Algebra, Springer, New York, 1988.

[12] M. Mitrović, S. Crvenković and D. A. Romano, Semigroups with apartness: constructive versions of some classical theorems, in: Proceedings of The 46th Annual Iranian Mathematics Conference, 25-28 August 2015, Yazd University, Yazd, Iran, 2016, 64-67.

[13] M. S. Rao and A. El-M. Badawy, Filters of lattices with respect to a congruence, Discuss. Math. Gen. Algebra Appl. 34(2014), 213-219.

[14] D. A. Romano, A note on a family of quasi-antiorder on semigroup, Kragujevac J. Math. 27 (2005), 11-18.

[15] D. A. Romano, The second isomorphism theorem on ordered set under anti-orders, Kragujevac J. Math. 30 (2007), 235-242.

[16] D. A. Romano, A note on quasi-antiorder in semigroup, Novi Sad J. Math. 37(1) (2007), 3-8.

[17] D. A. Romano, An isomorphism theorem for anti-ordered sets, Filomat 22(1) (2008), 145-160.

[18] D. A. Romano, On quasi-antiorder relation on semigroups, Mat. Vesnik 64(3) (2012), 190-199.

[19] D. A. Romano, Co-ideals and co-filters in ordered set under co-quasiorder, Bull. Int. Math. Virtual Inst. 8(1) (2018), 177-188.

[20] A. Troelstra and D. van Dalen, Constructivism in Mathematics, An Introduction, Volume II, North-Holland, Amsterdam, 1988.

${ }^{1}$ International Mathematical Virtual Institute,

6, KordunašKa Street, 78000 BAnJA LuKA,

Bosnia AND Herzegovina

Email address: bato49@hotmail.com 